\title{
Developmental Regulation of DNA-Topoisomerases during Drosophila Embryogenesis
}

\author{
Mark J . Gemkow, ${ }^{1}$ J oachim Dichter, and Donna J . Arndt-J ovin ${ }^{2}$ \\ Department of Molecular Biology, Max Planck Institute for Biophysical Chemistry, 37070 Göttingen, Germany
}

Type I and type II DNA-topoisomerases are essential enzymes that mediate replication, transcription, recombination, and mitosis in multicellular eukaryotes but the extent of their interchange for specific reactions in vivo is controversial. Expression patterns for topoisomerase I and topoisomerase II during the embryogenesis of Drosophila melanogaster were compared with patterns of DNA replication and expression of the histone genes. In late oogenesis the maternally supplied top2 mRNA was evenly distributed throughout the egg with elevated levels at the posterior tip, a pattern that is maintained in syncytial blastoderm embryos. During gastrulation, top2 mRNA became differentially localized only to regions of DNA replication, including new expression in the gonads preceding mitosis/meiosis. Significantly higher levels of top2 mRNA were found in mitotic compared to endoreplicating tissues. The total histone mR NA was exclusively associated with DNA replication but, in contrast to top2 mRNA, mitotic and endoreplicating cells contained similar expression levels with no expression in the gonads. Striking differences exist between the distribution of the top2 mRNA and topoisomerase II protein. The protein localizes to all evolving nuclei where it persists throughout embryogenesis. A high level of top1 mRNA transcript was present without differential tissue distribution throughout embryogenesis. ๑ 2001 Academic Press

Key Words: mRNA; histones; BrdU; topoisomerases; endoreplication; mitosis.

\section{INTRODUCTION}

In eukaryotes, two different types of DNA-topoisomerases have been found: type I and type II enzymes. Type I enzymes catalyze cleavage and religation reactions of DNA single strands whereas type II enzymes catalyze these reactions with double-stranded DNA

\footnotetext{
${ }^{1}$ Current address: Evotec NeuroSciences GmbH, Schnackenburgallee 114, 22525 Hamburg, Germany.

2 To whom correspondence and reprint requests should be addressed. Fax: +49-551-2011467. E-mail: djovin@mpc186.mpibpc. gwdg.de.
}

molecules. Topoisomerases alter the DNA topology and are necessary for releasing torsional stress in the DNA in processes such as replication and transcription; for review see [1]. In contrast to the prokaryotic enzymes, the eukaryotic topoisomerases are only able to reduce supercoiling. However, a supercoiling (gyrase) activity has been cloned from two eukaryotes $[2,3]$ which appears to function only in conjunction with topoisomerase II (TOPO II).

A number of studies have shown that TOPO II is an essential enzyme required for the decatenation of chromatids at mitosis [4-6]. The higher eukaryotes encode two genes for topoisomerase II (top2) [7] as well as process several splice site variants, suggesting additional roles and/or compartmentalization for the protein in multicellular organisms $[8,9]$. Although TOPO II may substitute for a top1 mutant in yeast [10], the same is not true in a multicellular organism where a top1 mutant leads to developmental arrest and lethality at blastoderm formation [11]. Thus, a clear specialization in function has been imposed on the enzymes in multicellular organisms.

The fact that TOPO II is found as a chromatin component of metaphase and meiotic chromosomes [12, 13] as well as isolated in the "nuclear matrix" fraction of extracted nuclei [14] has led to the postulate that TOPO II plays a structural as well as a functional role in chromatin assembly and modulation [15]. The enzyme shows preferential binding to a number of non-B DNA structures such as crossovers [16], supercoiled or Z-DNA [17, 18], and bent DNA [19], suggesting that regulation of nuclear processes might be mediated by topologically distinguishable states of the DNA. It has been postulated that lower eukaryotes (yeast and Drosophila) might regulate multiple TOPO II functions with a single gene through posttranslational enzyme modification, such as phosphorylation [20]. In vitro studies on Drosophila TOPO II indicate that ATP turnover is enhanced by phosphorylation of the enzyme by casein kinase II [21]. For the mammalian enzymes phosphorylation appears to be a regulator of enzyme stability and chromatin association rather than of the activity $[22,23]$. 
Detailed information on the distribution of mRNA and protein during Drosophila embryogenesis could help clarify the roles of both TOPO I and TOPO II in nuclear processes and organization. Whalen et al. [24] showed by blotting experiments that the mRNA and protein levels for TOPO II during embryogenesis were not strictly correlated. Recently, the level and control of expression of top1 mRNA during embryogenesis have been studied by blotting [25]. In this paper we present data on the distribution of mRNAs for topl, top2, and histones as well as for TOPO II protein throughout embryonic devel opment. We demonstrate a strong positive correlation between mitotically active cells and top2 mRNA transcription with considerably lower expression in endoreplicating tissues and complete absence in cells arrested for DNA replication. In contrast, the TOPO II protein persists in the nuclei of nondividing cells at a constant ratio to DNA content.

\section{MATERIALS AND METHODS}

Fly stocks and culture. The wild-type strain Oregon R-P2 [26] was used throughout. Larvae were raised in plastic bottles on a medium of cornmeal, agar, soy bean meal, malt extract, molasses, yeast, $0.5 \%(\mathrm{v} / \mathrm{v})$ propionic acid, and the mold inhibitor methyl phydroxybenzoate (Nipagin, Caesar and Lorentz, Hilden, FRG). Flies were held in a medium-size cage $\left(25 \times 20 \times 25 \mathrm{~cm}^{3}\right)$ at $22^{\circ} \mathrm{C}$. Eggs were collected on apple-juice agar plates containing fresh yeast.

mRNA in situ hybridization in whole mount embryos. The DNA probes for top1 and top2 were kindly provided by Dr. T.-s. Hsieh [27, 28]. The cDNA clone of the complete histone cluster was a kind gift from Dr. A. Udvardy [29]. Probes were labeled with digoxigenindUTP by random priming using the Roche High Prime kit. The probes were size checked on agarose gels and sonicated to yield a homogeneous length distribution which peaked between 200 and 300 bp; see [30] for details. Hybridization to mRNA in whole mount Drosophila embryos was essentially carried out as described by Tautz and Pfeifle [31]. This protocol includes a proteinase $\mathrm{K}$ digestion step in combination with a second fixation step. Different conditions of digestion and postfixation were evaluated to ensure reproducible accessibility of the probe to the target mRNA in all tissues. In situ signals were detected using polyclonal sheep Fab fragments against digoxigenin coupled to alkaline phosphatase (Roche) and color was developed with nitroblue tetrazolium and 5-bromo-4chloro-indolylphosphate.

Incorporation of BrdU into embryos and combination with mRNA in situ hybridization. The incorporation of bromodeoxyuridine (BrdU) was performed according to the literature [32]. After fixation and devitellinization, embryos were exchanged into PBTX (PBS + $0.05 \%$ Triton $\mathrm{X}-100$ ) and a regular in situ protocol was performed. The hybridization signal was detected as above. After development of the phosphatase reaction, the embryos were washed three times for 20 min with PBTX and the DNA was depurinated with $2 \mathrm{~N} \mathrm{HCl}$ for $45 \mathrm{~min}$. Embryos were thereafter washed with PBTX $(3 \times 30 \mathrm{~min})$ and incubated with monoclonal IgG against BrdU (Sigma, St. Louis, $\mathrm{MO})$ at $4^{\circ} \mathrm{C}$ overnight. After three washes the embryos were incubated at room temperature with a peroxidase-coupled goat-antimouse polyclonal IgG (Dianova). After washes the peroxidase reaction was performed in $100 \mathrm{mM}$ sodium phosphate buffer, $\mathrm{pH}$ 6.8, using 3,3'-diaminobenzidine $(0.5 \mathrm{mg} / \mathrm{ml})$ as color reagent.

Antibodies. Polyclonal antibodies were raised in rabbits against our most highly purified fraction of DNA-topoisomerase II [17] and IgG fractions prepared by Sepharose G chromatography. In some cases affinity-purified fractions were prepared by chromatography on a TOPO II column (AffiGel-10; Bio-Rad). The antibody shows monospecific binding to TOPO II by Western blotting of embryo extracts [17]. In addition the antibody inhibits TOPO II enzymatic activity both in vivo [6] and in vitro [17]. Whole mount stainings have been already described [6]. This antibody was conjugated with the succinimidylester Cy-3-OSu (Cy3, Amersham) as described previously [33].

Topo II protein staining in whole mount embryos. Fixation of embryos was described earlier [30] using Burgonye's buffer [34]. Blocking: $1 \%$ bovine serum albumin $60 \mathrm{~min}$ at room temperature in PBT (PBS + 0.1\% Tween 20); antibody incubation: polyclonal anti TOPO II (W4) coupled with Cy3 overnight at $4^{\circ} \mathrm{C}$ in PBT. Embryos were washed four times for 20 min each with PBT. The DNA was counterstained with $3 \mu \mathrm{M}$ 4,6-diamidino-2-phenylindole. Embryos were exchanged into Mowiol 4-88 (Hoechst) via a step gradient and mounted for inspection and documentation. I dentical distributions of the protein in Drosophila were obtained on cryosections performed as controls for penetrability of the antibody (data not shown).

Microscopy. The histochemically stained embryos were analyzed with a Zeiss Axiophot equipped with Nomarski-optics. Overviews were taken with a Plan-Neofluar 20x/0.5 and details with a PlanApochromat $63 \times / 1.4$ oil immersion. Images were recorded with a Kontron ProgRes 3012 camera via a plugin provided by Kontron directly into Adobe Photoshop.

Fluorescence microscopy. The fluorescent images shown in this paper were recorded with a Zeiss LSM 310 confocal laser scanning microscope and are presented in an "extended focus" fashion, composed in SCIL-Image (TNO Institute of Applied Physics, TU Delft, The Netherlands). Images represent an overlay of 10 to 15 confocal sections taken with an interval of $1 \mu \mathrm{m}$. I mages were taken with a Plan-Neofluar 25x/0.8 water-oil lens. For the intensity measurements presented in Fig. 2, fields with an approximated size of 700 $\mu \mathrm{m}^{2}$ were taken with a Plan-N eofluar $40 \mathrm{x} / 1$.3 oil immersion lens. For excitation of the Cy3-labeled embryos an argon-ion-laser with a wavelength of $514 \mathrm{~nm}$ was used and a longpass emission filter of 575 $\mathrm{nm}$.

Image processing. Fluorescent images were composed as overlays using SCIL-Image (TNO Institute of Applied Physics). Measurements of integrated intensities in fluorescent images were performed in NIH Image 1.61. The figures were composed with Photoshop 5.0 and FreeHand 8.0 (Adobe Systems, Mountain View, CA; Macromedia).

\section{RESULTS}

\section{Distribution of DNA-Topoisomerase II mRNA and Protein during Embryogenesis}

The embryogenesis of Drosophila starts with rapid nuclear divisions before cell membranes are established and zygotic transcription begins. Both TOPO II protein and mRNA are maternally stored in the egg. Top2 mRNA is present throughout the egg in late stages of oogenesis with a slightly higher concentration at the posterior tip (see Fig. 1A, arrow in the magnification). This distribution is stable in the embryo until pole buds arise (compare Figs. 1B and 1D), after which the zygotic message takes on a more uniform distribution which persists until the end of germband elongation. At germband retraction (stage 12) the top2 mRNA begins to localize to the ventral parts of the embryo. The top2 mRNA persists in the nervous system and is 


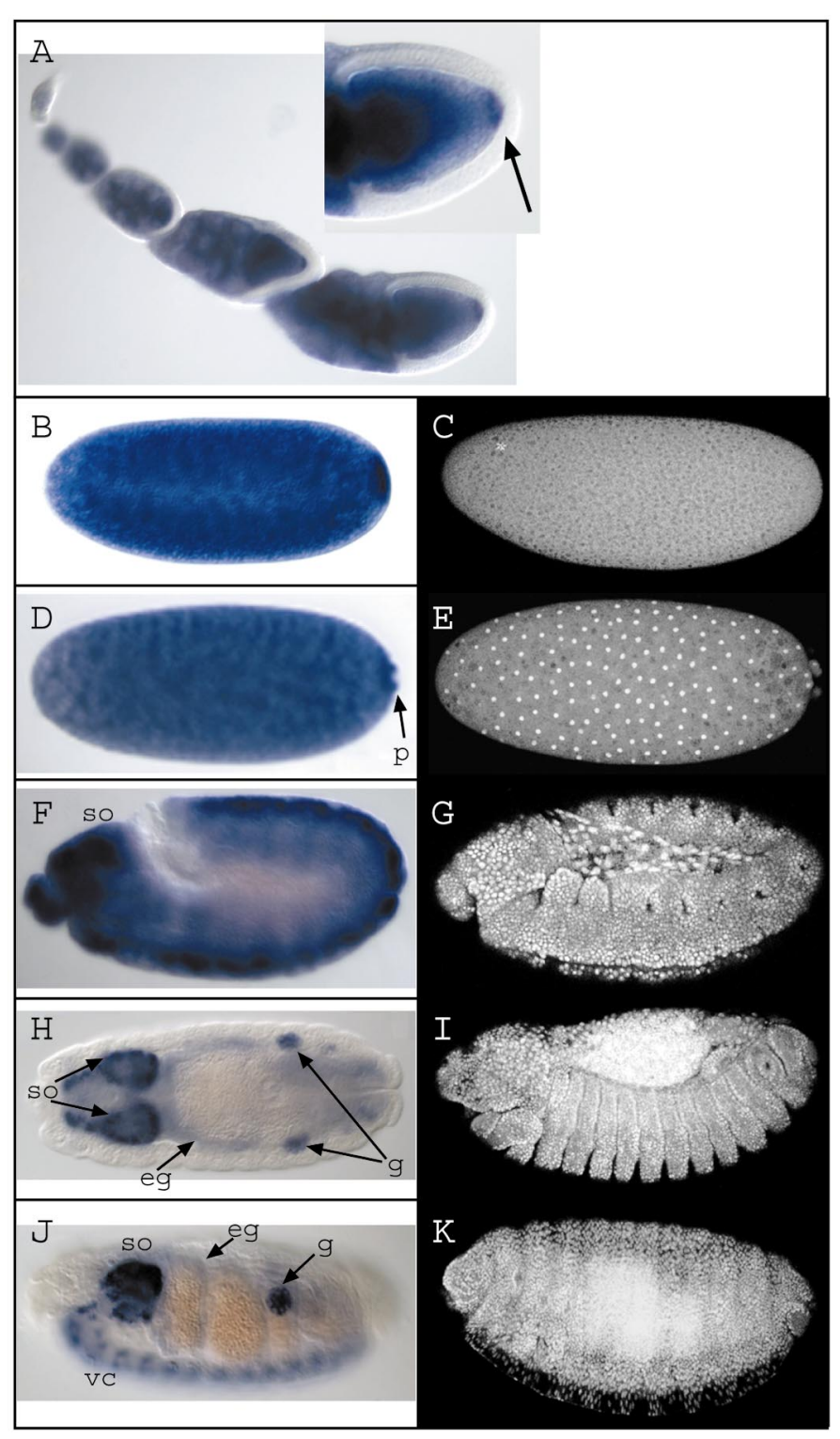

FIG. 1. Comparison of mRNA and protein distribution of DNA-topoisomerase II during embryogenesis. On the left side the distribution of the mRNA is shown by hybridization with the complete top2 cDNA labeled with digoxigenin and detected with an alkaline-phosphatase-coupled antibody indicated by the blue color. On the right grayscale fluorescent images of the TOPO II protein distribution detected with a Cy3-coupled polyclonal antibody are shown. A: mRNA localization in oocytes; in stage 10 oocytes elevated levels are detectable at the posterior tip, see arrow in the inset. $B$ and $C$ : Stage 1 embryos. $D$ and $E$ : Stage 3 embryos; in $E$ division cycle 9 is shown. $F$ and $G$ : Stage 11 embryos. $\mathrm{H}$ and I: Stage 13 embryos; the embryo in $\mathrm{H}$ is shown from dorsal. J and K: Stage 16 embryos. Note that the tan coloring in the gut in the late stages is due to autofluorescence and nonspecific binding of the secondary antibody. This nonspecific staining is more pronounced in the late stages than in the early blastoderm stages. eg, endoreplicating gut epithelium; g, gonads; p, pole cells; so, supraesophagial ganglia; vc, ventral cord (these abbreviations are valid for all figures). lost in more and more tissues. By stage 13 the mRNA can only be detected in the ventral cord, the supraesophagial ganglia, and the gonads and in lower concentration in the endoreplicating gut epithelium and malpighian tubules (Fig. $1 \mathrm{H}$ for stage 13 and $1 \mathrm{~J}$ for stage 16). (As indicated under Materials and Methods section in situ probe length was reduced to 200-300 bp by sonication as described previously [30] to improve probe penetration for all hybridizations.)

TOPO II protein was detectable by immunofluorescence in all regions and tissues throughout embryonic development. The embryogenesis starts with a vast pool of maternally supplied protein [shown by Western blotting, 24, 35] which is evenly distributed throughout the syncytial blastoderm (Fig. 1C). The protein is rapidly titrated out of the cytoplasm by ongoing DNA synthesis and becomes localized to the DNA as more potential binding loci on the chromatin arise (Fig. 1E). By comparing the signal intensities of single confocal sections in the early nuclear division cycles, we find that the ratio between cytoplasmic and nuclear TOPO II protein equals 1 until cycle 5 and drops to around 0.2 in cycle 14 embryos prior to gastrulation (see Fig. 2 for measurements between cycles 6 and 14). These findings are consistent with the data from $\mathrm{N}$ olan et al ., who showed by Western blots of homogenized embryos that the ratio between nuclear TOPO II and cytoplasmic TOPO II increases in late embryogenesis [35].

\section{Comparison of top2 mRNA Distribution with DNA Synthesis}

Bromodeoxyuridine incorporation may be used as a marker of DNA synthesis during Drosophila embryo-

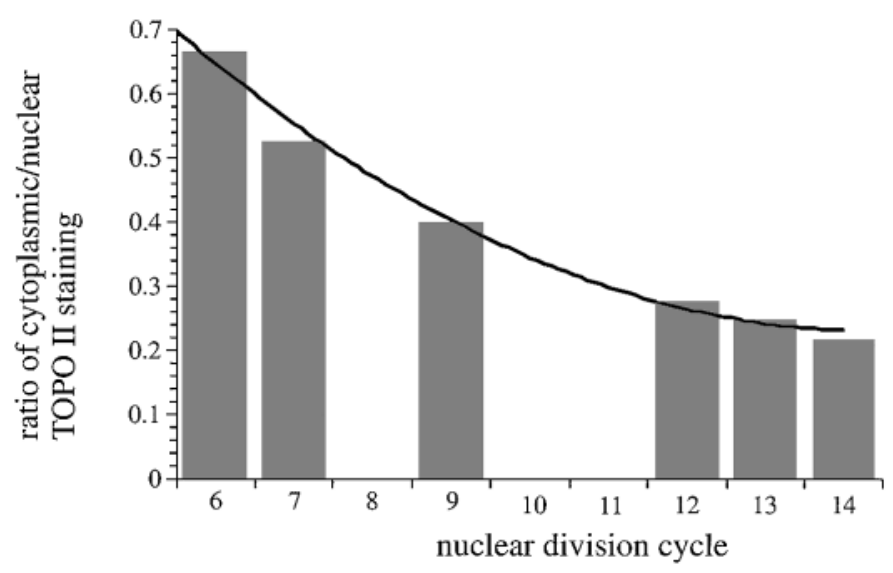

FIG. 2. Ratio between TOPO II protein in the cytoplasm and the nuclei in early nuclear division cycles. Measurements were made in single optical sections and mean intensity values of at least 10,000 pixels were taken from the data sets taken as described under Material and Methods. Measurements shown were made in cycles 6 , $7,9,12,13$, and 14 . With increasing amounts of DNA and nuclei, the protein is titrated out of the cytoplasm. 


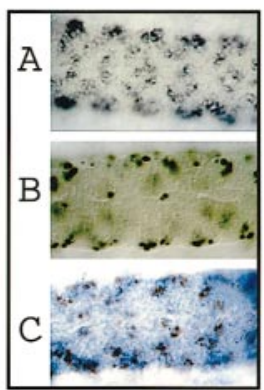

3

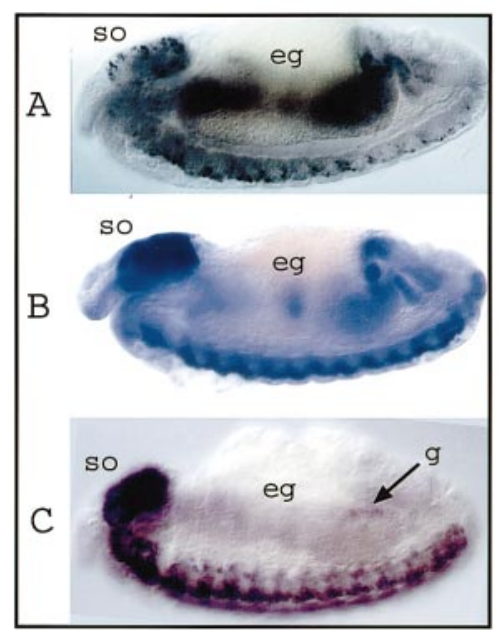

4

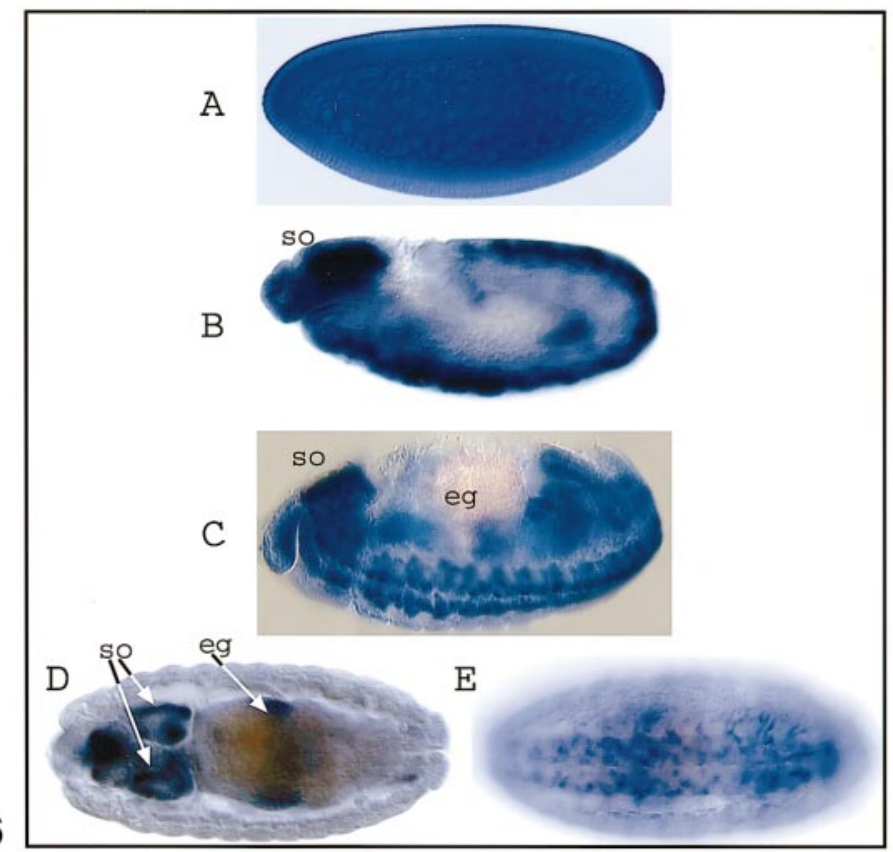

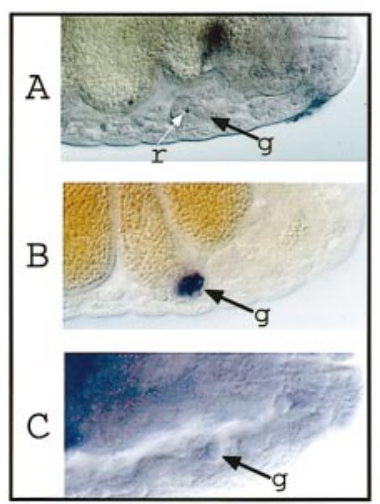

5

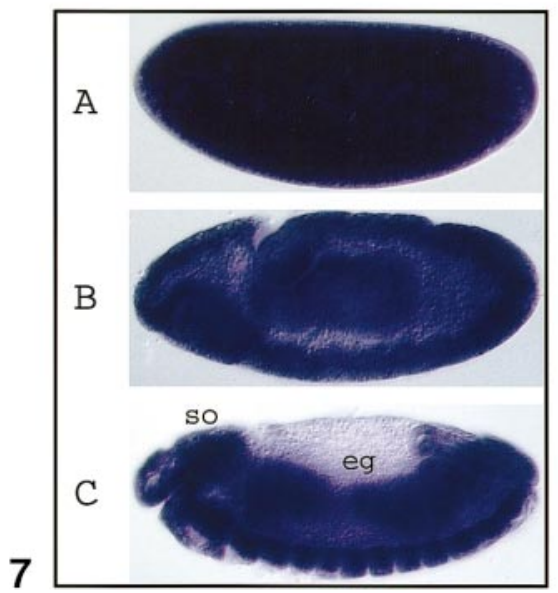

FIG. 3. Comparison of top 2 mRNA expression, shown by phosphatase staining in blue, and BrdU incorporation, shown by peroxidase staining in brown, in the CNS (ventral cord) of stage 13 embryos. A: top2 mRNA expression; B: BrdU incorporation; C: combination of the two techniques. Despite the higher blue background, the signals are seen to colocalize.

FIG. 4. Comparison between BrdU and mRNA distributions of top 2 and the complete histones in a stage 13 embryo. A: BrdU incorporation. B: Histone mRNA distribution, the expression parallels the BrdU incorporation. C: top2 mRNA distribution; the expression in the endoreplicating tissues is lower than in mitotic cells.

FIG. 5. Comparison between BrdU incorporation (brown) and mRNA distributions (blue) in gonads of late stage. A: BrdU incorporation; $r$, single replicating nucleus. B: top2 mRNA. C: histone mRNA: no signal above the background is detectable even after prolonged enzymatic development.

FIG. 6. Distribution of complete histone mRNA during embryogenesis shown by hybridization using digoxigenin-labeled cDNA of the histone gene cluster. Probe localization was detected by alkaline phosphatase staining shown in blue. The expression is closely coupled to DNA synthesis. A: Cellular blastoderm stage. B: Stage 11 embryo; the mRNA is diminished in parts of the embryo but remains in the CNS (ventral cord). C: Stage 12/13 embryo; the mRNA is mainly expressed in the CNS and the endoreplicating gut; see also Fig. 4B. D: Stage 13 embryo shown from dorsal; expression levels in the supraesophagial ganglia are comparable to the midgut. E: Same embryo as D from the ventral side focused on the CNS; the mRNA is strongly expressed in cells that are mitotic; for comparison see also Fig. 3.

FIG. 7. Distribution of the topoisomerase I mRNA during embryogenesis. The mRNA was hybridized with the complete topl cDNA labeled with digoxigenin and detected by alkaline phosphatase staining shown in blue. The topl mRNA is expressed throughout embryogenesis in all embryonic tissues with no obvious quantitative differences. A: blastoderm; B: stage 9; C: stage 13. 
genesis [32]. We used this method to compare the replication patterns with top2 mRNA distribution during embryogenesis. Data are shown for gastrulation times after the cessation of synchronous replication. We found strong positive correlations between the BrdU incorporation and the localization of the top2 mRNA in the supraesophageal ganglia and in the ventral cord after the 15th cell cycle. In the endoreplicating gut epithelium, top2 mRNA was detectable but at a much lower level than that seen in the mitotic cells (compare Figs. 4A and 4C). By double staining for BrdU incorporation and mRNA in situ hybridization, we were able to show that all mitotic cells lie within the top2 expression boundaries (see Figs. $3 \mathrm{~A}$ and $3 \mathrm{C}$ ). A striking difference was found in the gonads where top 2 mRNA was detectable from stage 13 on, although mitosis starts at the earliest in $16-\mathrm{h}$-old embryos $\left(25^{\circ} \mathrm{C}\right)$, which equals a late stage 16 embryo [36; Fig. 5]. Ther efore top2 mRNA expression clearly precedes mitosis in the gonads.

\section{Comparison of the top2 mRNA Distribution with the \\ Total Histone mRNA Distribution}

Another direct measure of new DNA synthesis in both mitotic and endoreplicating cells is given by histone gene transcription (see Figs. 4 and 6). We investigated how closely the mRNA for histone correlated with DNA replication in both types of cells. Our hybridization probe covered the whole histone gene cluster [29]. In contrast to the results for top2 mRNA, we observed no significant difference between the amount of total histone mRNA in the ventral cord (mitotic) and the gut epithelium (endoreplicative) (Figs. 4B and 6C). For comparison, top2 mRNA is shown for the same stage (Fig. 4C). Also, contrary to our findings for top2 mRNA there was no histone mRNA detectable in the gonads. A comparison of the gonadal region of the embryo at higher magnification probed for BrdU incorporation (A), top2 mRNA (B), and histone mRNA (C) is shown in Fig. 5.

\section{Distribution of the top1 mRNA during Embryogenesis}

Top1 mRNA was found to persist in all cells throughout embryogenesis. As in the case of top2 mRNA, embryogenesis begins with maternally supplied top1 mRNA, distributed evenly throughout the syncytial blastoderm (Fig. 7A), and becomes incorporated into the evolving cells. A high level of topl mRNA was found in all cells throughout embryogenesis with no differential distribution (see Fig. 7). This expression pattern of top1 is consistent with the high transcriptional activity of all tissues during embryogenesis and corroborates the data of Brown et al . [25], who observed strong zygotic transcription by Northern blotting throughout embryogenesis. High amounts of top1 mRNA are also detectable in the endoreplicating gut tissue. We assume that these results reflect primarily the role of the enzyme in transcription of genes necessary for morphogenesis, but we cannot rule out a direct involvement of the enzyme in endoreplication.

\section{DISCUSSION}

We have investigated the distribution of both TOPO II protein and mRNA during Drosophila melanogaster embryogenesis using whole mount in situ hybridization and immunocytochemistry and compared these patterns with those for mRNAs from topl and histones. The pattern of maternally supplied top2 mRNA in late oogenesis was maintained in syncytial blastoderm embryos where it was found throughout the embryo with elevated levels at the posterior tip (Fig. 1A). This differential distribution was lost at the time pole buds developed. However, a new distribution appeared during gastrulation stages, where top2 mRNA was restricted to regions of the embryo involved in DNA synthesis (Figs. $1 \mathrm{H}, 1 \mathrm{~J}, 3 \mathrm{~A}$, and $4 \mathrm{C}$ ). In addition, top2 mRNA was detected in the gonads preceding mitosis. From our analysis we cannot distinguish between new expression in the gonads or persistent sequestration of the mRNA into the pole cells as has been described for the cyclin B message [37]. Our data explain the earlier observations from whole embryo extracts and blotting on the strikingly different levels of top1 and top2 mRNAs during embryogenesis [25, 38] and differentiate the specific distribution in the tissues of the embryo.

Our more detailed analysis of the top2 mRNA distribution in relationship to DNA replication and histone mRNA (Fig. 4) supports the thesis that top2 expression is restricted to replicating cells (Fig. 3). However, the large differences in the levels of top2 mRNA in mitotic versus endoreplicating cells (Figs. 4, $1 \mathrm{H}$, and 1J ) which we have documented shows that it is not replication per se which triggers the high mRNA transcription. We and others have shown that TOPO II is absolutely essential for the separation of chromatids at mitosis [6, 39]. Our in vivo inhibition studies [6] as well as experiments on in vitro chromatin reconstitution [40] demonstrated that TOPO II plays an additional role in the condensation of chromatin at metaphase, although $70 \%$ of the enzyme associated with the DNA at interphase is lost from the chromosomes during mitosis [41].

The data presented here indicate a similar rate of DNA replication in endoreplicating compared with mitotic cells as determined by the rate of bromodeoxyuridine incorporation and by histone mRNA expression (Figs. 4 and 6 and data not shown). The large difference we see in top2 mRNA levels in the two tissues implies that TOPO II is probably not directly responsible for relaxation of supercoiling of DNA during rep- 
lication, but rather this function may be performed exclusively by TOPO I. The data underscore that the primary role of the newly expressed enzyme is for chromosome decatenation and chromatid separation and suggest that considerably smaller amounts of the enzyme are required as a structural component of newly synthesized chromatin.

The distinct increase in top2 mRNA we observed in the gonads (Fig. 5) before significant replication ensues supports a specific role for the enzyme in meiotic chromosome recombination. Rose et al. [42] demonstrated an arrest at meiosis for top2 yeast mutants but the exact step where TOPO II is required has not yet been determined.

We found production of top1 mRNA in all cells throughout embryogenesis (Fig. 7), which is consistent with the presumed role of this DNA topoisomerase in transcription. Lee et al. [11] demonstrated that a deletion mutant of Drosophila topl was lethal after blastoderm formation, a time when strong zygotic transcription is initiated. Recently Shaiu and Hsieh [43] found that TOPO I is specifically targeted to active RNA polymerase II sites by its N-terminal domain. Our data show the disappearance of top2 mRNA from nonreplicating cells in the embryo. The continued presence of the enzyme in all nuclei is obviously not sufficient for a functional rescue of topl mutants in Drosophila, as is the case in yeast [10]. These observations raise the possibility that only newly synthesized TOPO II is enzymatically active and the TOPO II protein bound to chromatin in arrested cells is purely structural in nature, or that active TOPO II requires posttranslation modification or targeting.

There are clearly different roles for the two mammalian TOPO II enzymes although their intracellular distribution in various cell types and under various growth conditions are disputed [15, 44-47]. Since top2 is a single copy gene in Drosophila [28] and no evidence has been found for differential splicing of the mRNA, posttranslation modification of the protein during embryogenesis might either target the enzyme to metaphase or stabilize a structural form of the enzyme. There are conflicting data in the literature concerning the role of phosphorylation on TOPO II. Phosphorylation appears to change the activity of the yeast enzyme $[48,49]$. In contrast, studies on Drosophila TOPO II C-terminal truncation mutants which abolish the phosphorylation sites remained active in vitro and complement yeast top2 mutants [50]. On the other hand Corbett et al. [21] showed that the phosphorylated enzyme has a higher ATP turnover rate in vitro. Recent studies with the mammalian TOPO II $\beta$ showed identical in vitro enzymatic activity for phosphorylated and dephosphorylated forms but dramatically different DNA binding properties [51]. Another group has reported a nonphosphorylating modification of TOPO II $\alpha$ by ERK2 [52]. Although a recent paper purports to demonstrate changes in nucleic acid affinity of Drosophila TOPO II directed by mitotic kinases [53], the data should be viewed with caution since the authors' protocol for isolation of mitotic cells results in cells of abnormal metabolic state (our unpublished observations.) At the moment there are no reliable data available for the extent and nature of the posttranslational modifications of Drosophila TOPO II during embryogenesis.

From our data we conclude that there appears to be a fraction of Drosophila TOPO II that undergoes a fast turnover in mitotically active cells and that is reflected by the mRNA expression profile of the top 2 gene. That this expression is linked to mitosis and does not solely reflect the increase in DNA content is clear by comparing mitotic and endoreplicating tissues. The TOPO II protein bound to chromatin appears to be much more stable, as no decrease in protein levels could be detected by immunofluorescence quantitation from the beginning of cycle 15 to the end of embryogenesis in nonreplicating cells (data not shown).

Our data support the singular role of topoisomerase I in controlling superhelical density during transcription.

\section{REFERENCES}

1. Wang, J. C. (1996). DNA topoisomerases. Ann. Rev. Biochem. 65, 635- 692.

2. Kobayashi, M., Aita, N., Hayashi, S., Okada, K., Ohta, T., and Hirose, S. (1998). DNA supercoiling factor localizes to puffs on polytene chromosomes in Drosophila melanogaster. Mol. Cell Biol. 18, 6737- 6744.

3. Ohta, T., Kobayashi, M., and Hirose, S. (1995). DNA supercoiling factor reveals a distinctive $\mathrm{Ca}^{2+}$-binding protein. J. Biol. Chem. 270, 15571-15575.

4. Holm, C., Stearns, T., and Botstein, D. (1989). DNA topoisomerase II must act at mitosis to prevent nondisjunction and chromosome breakage. Mol. Cell. Biol. 9, 159-168.

5. Clarke, D. J ., J ohnson, R. T., and Downes, C. S. (1993). Topoisomerase II inhibition prevents anaphase chromatid segregation in mammalian cells independently of the generation of DNA strand breaks. J . Cell Sci. 105, 563-569.

6. Buchenau, P., Saumweber, H., and Arndt-J ovin, D. J . (1993). Consequences of topoisomerase II inhibition in early embryogenesis of Drosophila revealed by in vivo confocal laser scanning microscopy. J . Cell Sci. 104, 1175-1185.

7. Austin, C. A., Sng, J. H., Patel, S., and Fisher, L. M. (1993). Novel HeLa topoisomerase II is the II beta isoform: Complete coding sequence and homology with other type II topoisomerases. Biochim. Biophys. Acta 1172, 283-291.

8. Davies, S. L., J enkins, J . R., and Hickson, I. D. (1993). Human cells express two differentially spliced forms of topoisomerase II beta mRNA. Nucleic Acids Res. 21, 3719-3723.

9. Zandvliet, D. W., Hanby, A. M., Austin, C. A., Marsh, K. L., Clark, I. B., Wright, N. A., and Poulsom, R. (1996). Analysis of foetal expression sites of human type II DNA topoisomerase alpha and beta mRNAs by in situ hybridisation. Biochim.Biophys. Acta 1307, 239-247. 
10. Goto, T., and Wang, J. C. (1985). Cloning of yeast TOP1, the gene encoding DNA topoisomerase I, and construction of mutants defective in both DNA topoisomerase I and DNA topoisomerase II. Proc. Natl. Acad. Sci. USA 82, 7178-7182.

11. Lee, M. P., Brown, S. D., Chen, A., and Hsieh, T. S. (1993). DNA topoisomerase I is essential in Drosophila melanogaster. Proc. Natl. Acad. Sci. USA 90, 6656-6660.

12. Hock, R., Carl, M., Lieb, B., Gebauer, D., and Scheer, U. (1996). A monoclonal antibody against DNA topoisomerasell labels the axial granules of Pleurodeles lampbrush chromosomes. Chromosoma 104, 358-366.

13. Earnshaw, W. C., Halligan, B., Cooke, C. A., Heck, M. M., and Liu, L. F . (1985). Topoisomerase II is a structural component of mitotic chromosome scaffolds. J . Cell Biol. 100, 1706-1715.

14. Berrios, M., Osheroff, N., and Fisher, P. A. (1985). In situ Iocalization of DNA topoisomerase II, a major polypeptide component of the Drosophila nuclear matrix fraction. Proc. Natl. Acad. Sci. USA 82, 4142- 4146.

15. Warburton, P. E., and Earnshaw, W. C. (1997). Untangling the role of DNA topoisomerase II in mitotic chromosome structure and function. BioEssays 19, 97-99.

16. Zechiedrich, E. L., and Osheroff, N. (1990). Eukaryotic topoisomerases recognize nucleic acid topology by preferentially interacting with DNA crossovers. EMBO J . 9, 4555- 4562.

17. Arndt-J ovin, D. J ., Udvardy, A., Garner, M. M., Ritter, S., and J ovin, T. M. (1993). Z-DNA binding and inhibition by GTP of Drosophila topoisomerase II. Biochemistry 32, 4862- 4872.

18. Glikin, G. C., J ovin, T. M., and Arndt-J ovin, D. J . (1991). Interactions of Drosophila DNA topoisomerase II with lefthanded Z-DNA in supercoiled minicircles. Nucleic Acids Res. 19, 7139-7144.

19. Bechert, T., Diekmann, S., and Arndt-J ovin, D. J . (1994). Human $170 \mathrm{kDa}$ and $180 \mathrm{kDa}$ topoisomerases II bind preferentially to curved and left-handed linear DNA. J . Biomol. Struct. Dynam. 12, 605- 623.

20. Dang, Q., Alghisi, G. C., and Gasser, S. M. (1994). Phosphorylation of the C-terminal domain of yeast topoisomerase II by casein kinase II affects DNA-protein interaction. J . Mol. Biol. 243, 10-24.

21. Corbett, A. H., DeVore, R. F., and Osheroff, N. (1992). Effect of casein kinase II-mediated phosphorylation on the catalytic cycle of topoisomerase II. Regulation of enzyme activity by enhancement of ATP hydrolysis. J. Biol. Chem. 267, 2051320518.

22. Burden, A. D., and Sullivan, D. M. (1994). Phosphorylation of the alpha- and beta-isoforms of DNA topoisomerase II is qualitatively different in interphase and mitosis in chinese hamster ovary cells. Biochemistry 33, 14651-14655.

23. Kimura, K., Nozaki, N., Enomoto, T., Tanaka, M., and Kikuchi, A. (1996). Analysis of M phase-specific phosphorylation of DNA topoisomerase II. J . Biol. Chem. 271, 21439-21445.

24. Whalen, A. M., McConnell, M., and Fisher, P. A. (1991). Developmental regulation of Drosophila DNA topoisomerase II. J . Cell Biol. 112, 203-213.

25. Brown, S. D., Zhang, C. X., Chen, A. D., and Hsieh, T. S. (1998). Structure of the Drosophila DNA topoisomerase I gene and expression of messages with different lengths in the 3' untranslated region. Gene 211, 195-203.

26. Allis, C. D., Waring, G. L., and Mahowald, A. P. (1977). Mass isolation of pole cells from Drosophila melanogaster. Dev. Biol. 56, 372-381.

27. Hsieh, T. S., Brown, S. D., Huang, P., and Fostel, J . (1992). Isolation and characterization of a gene encoding DNA topo- isomerase I in Drosophila melanogaster. Nucleic Acids Res. 20, 6177- 6182.

28. Wyckoff, E., Natalie, D., Nolan, J. M., Lee, M., and Hsieh, T. S. (1989). Structure of the Drosophila DNA topoisomerase II gene, nucl eotide sequence, and homology among topoisomerases II. J . Mol. Biol. 205, 1-14.

29. Lifton, R., Goldberg, M., Karp, R., and Hogness, D. (1977). The organization of the histone genes in Drosophila melanogaster: Functional and evolutionary implications. Cold Spring Harbor Symp. 43, 1047-1051.

30. Gemkow, M. J ., Verveer, P. J ., and Arndt-J ovin, D. J . (1998). Homologous association of the Bithorax-complex during embryogenesis: Consequences for transvection in Drosophila melanogaster. Devel opment 125, 4541- 4552.

31. Tautz, D., and Pfeifle, C. (1989). A non-radioactive in situ hybridisation method for the localization of specific RNAs in Drosophila embryos reveals translational control of the segmentation gene hunchback. Chromosoma 98, 81- 85.

32. Bodmer, R., Carretto, R. A., and Nung J an, Y. (1989). Neurogenesis of the peripheral nervous system in Drosophila embryos: DNA replication patterns and cell lineages. Neuron 3, 21-32.

33. Buchenau, P., Saumweber, H., and Arndt-J ovin, D. J . (1997). The dynamic nuclear redistribution of an hnRNP K-homologous protein during Drosophila embryo development and heat shock. Flexibility of transcription sites in vivo. J . Cell Biol. 137, 291303.

34. Burgonye, L. A., Waqar, M. A., and Atkinson, M. R. (1970). Calcium-dependent priming of DNA synthesis in isolated rat liver nuclei. Biochem. Biophys. Res. Commun. 39, 254-259.

35. Nolan, J. M., Lee, M. P., Wyckoff, E., and Hsieh, T. S. (1986). I solation and characterization of the gene encoding Drosophila DNA topoisomerase II. Proc. Natl. Acad. Sci. USA 83, 36643668.

36. Sonnenblick, B. P. (1941). Germ cell movements and sex differentiation of the gonads in the Drosophila embryo. Proc. Natl. Acad. Sci. USA 27, 484- 489.

37. Dalby, B., and Glover, D. M. (1993). Discrete sequence elements control posterior pole accumulation and translational repression of maternal cyclin B RNA in Drosophila. EMBO J. 12, 1219-1227.

38. Fairman, R., and Brutlag, D. (1988). Expression of the Drosophila type II topoisomerase is developmentally regulated. Biochemistry 27, 560-565.

39. Holm, C., Goto, T., Wang, J . C., and Botstein, D. (1985). DNA topoisomerase II is required at the time of mitosis in yeast. Cell 41, 553-564.

40. Adachi, Y., Luke, M., and Laemmli, U. K. (1991). Chromosome assembly in vitro: Topoisomerase II is required for condensation. Cell 64, 137-148.

41. Swedlow, J. R., Sedat, J . W., and Agard, D. A. (1993). Multiple chromosomal populations of topoisomerase II detected in vivo by time-lapse, three-dimensional wide-field microscopy. Cell 73, 97-108.

42. Rose, D., Thomas, W., and Holm, C. (1990). Segregation of recombined chromosomes in meiosis I requires DNA topoisomerase II. Cell 60, 1009-1018.

43. Shaiu, W. L., and Hsieh, T. S. (1998). Targeting to transcriptionally active loci by the hydrophilic $\mathrm{N}$-terminal domain of Drosophila DNA topoisomerase I. Mol. Cell. Biol. 18, 43584367.

44. Hadlaczky, G., Praznovszky, R., Sofi, J., and Udvardy, A. (1988). Intracellular forms of Drosophila topoisomerase II de- 
tected with monoclonal antibodies. Nucleic Acids Res. 16, 10013-10023.

45. Heller, R., Shelton, E., Dietrich, V., Elgin, S., and Brutlag, D. (1986). Multiple forms and cellular localization of Drosophila DNA topoisomerase II. J . Biol. Chem. 261, 8063- 8069.

46. Watanabe, M., Tsutsui, K., Tsutsui, K., and Inoue, Y. (1994). Differential expressions of the topoisomerase II alpha and II beta mRNAs in developing rat brain. Neurosci. Res. 19, 51-57.

47. Chen, J . L., and Longo, F. J . (1996). Expression and localization of DNA topoisomerase II during rat spermatogenesis. Mol. Re prod. Dev. 45, 61-71.

48. Alghisi, G. C., Roberts, E., Cardenas, M. E., and Gasser, S. M. (1994). The regulation of DNA topoisomerase II by casein kinase II. Cell. Mol. Biol. Res. 40, 563-571.

49. Cardenas, M. E., and Gasser, S. M. (1993). Regulation of topoisomerase II by phosphorylation: A role for casein kinase II. J . Cell Sci. 104, 219-225.

Received J uly 3, 2000

Revised version received September 13, 2000
50. Crenshaw, D. G., and Hsieh, T. (1993). Function of the hydrophilic carboxyl terminus of type II DNA topoisomerase from Drosophila melanogaster II. In vivo studies. J . Biol. Chem. 268, 21335-21343.

51. Kimura, K., Saijo, M., Tanaka, M., and Enomoto, T. (1996). Phosphorylation-independent stimulation of DNA topoisomerase II alpha activity. J . Biol. Chem. 271, 10990-10995.

52. Shapiro, P. S., Whalen, A. M., Tolwinski, N. S., Wilsbacher, J ., Froelich-Ammon, S. J ., Garcia, M., Osheroff, N., and Ahn, N. G. (1999). Extracellular signal-regulated kinase activates topoisomerase II alpha through a mechanism independent of phosphorylation. Mol. Cell. Biol. 19, 3551-3560.

53. Rzepecki, R., and Fisher, P. (2000). During both interphase and mitosis, DNA topoisomerase II interacts with DNA as well as RNA through the protein's C terminal domain. J . Cell Sci. 113, 1635- 1647. 